\title{
A Geo-Location based Mobile Service that Dynamically Locates and Notifies the nearest Blood Donors for Blood Donation during Medical Emergencies
}

\author{
Saurin Parikh \\ Assistant Professor \\ Department of Computer \\ Science and Eng. \\ Institute of Technology, Nirma \\ University, Ahmedabad
}

\author{
Preeti Kathiria \\ Assistant Professor \\ Department of Computer \\ Science and Eng. \\ Institute of Technology, Nirma \\ University, Ahmedabad
}

\author{
Yashesh Vaghela, Harit \\ Shah, Darshan Dholakiya \\ Students \\ Department of Computer \\ Science and Eng. \\ Institute of Technology, Nirma \\ University, Ahmedabad
}

\begin{abstract}
Medical emergencies arise at any time or anywhere. During medical emergencies blood transfusion may be required to treat patients. The hospitals and clinics especially in Indian rural areas find it difficult to obtain blood instantaneously, especially of negative or rare blood group types [1]. The problem is more severe, when life is lost due to unavailability of blood supply. To solve this, several solutions have been proposed by various Authors; wherein the blood donor's static contact details is maintained by several agencies or blood banks [2]. However these solutions' were not adequate, as it provides list of donors based on the static contact location of the donors, as provided during registration. Whereas in real scenario, the actual location of the donor could be far away from the patient's site. This paper has proposed a system for dynamically locating and notifying the nearest donors for blood requirement. The system tracks the location of donors (volunteers) through GPS or Mobile Network Location and allows requestor to search nearest donors based on their actual location at the time of need. This proposed solution provides a reliable method of locating Blood donors especially in distant rural areas, where few blood banks are available.
\end{abstract}

\section{General Terms}

Geo-Location Based tracking, Global positioning system (GPS) and Mobile Network location based Tracking, Personal location based Alerting System, Mobile Rescue System and SMS based service.

\section{Keywords}

Mobile Application to locate nearest Blood Donors, GPS based tracking of Blood Donors' Geo-location, Android based solution to find nearby Blood Donors, and SMS based Help Confirmation.

\section{INTRODUCTION}

India faces a blood deficit of approximately 30-35 percent annually. The country needs around eight to 10 million units of blood every year but manages an approximately 5.5 million units [1]. The Ministry of Health and Family Welfare as on November 2012, states that there are 2556 licensed blood banks in India with 1525 private blood banks and 981 government blood banks [2]. A detailed zone-wise analysis of the availability of blood banks in India is shown in Table 1. It shows that Blood banks are not located uniformly in all parts of the country. In regions where there are fewer blood banks, there is an acute need to locate donors at the time of medical emergencies. Some websites register blood donors and provide static contact data of donors; however these sites do not dynamically provide details about nearest donors based on their actual location at time of need [3].

TABLE I: ZONE WISE BLOOD BANKS IN INDIA [2]

\begin{tabular}{|c|c|c|c|}
\hline Zone & $\begin{array}{c}\text { Government } \\
\text { Blood } \\
\text { Banks }\end{array}$ & $\begin{array}{c}\text { Private } \\
\text { Blood } \\
\text { Banks }\end{array}$ & $\begin{array}{c}\text { Total } \\
\text { Blood } \\
\text { Banks }\end{array}$ \\
\hline North Zone & 229 & 272 & 501 \\
\hline South Zone & 227 & 690 & 917 \\
\hline West Zone & 173 & 373 & 546 \\
\hline East Zone & 204 & 100 & 304 \\
\hline $\begin{array}{c}\text { North-East } \\
\text { Zone }\end{array}$ & 75 & 33 & 108 \\
\hline $\begin{array}{c}\text { Central Zone } \\
\text { Union } \\
\text { Territories }\end{array}$ & 71 & 106 & 177 \\
\hline Total & 981 & 1575 & 2556 \\
\hline
\end{tabular}

This Proposed System on behalf of the requester (patient) locates the nearest blood donors dynamically based on their actual geo-location and automatically notifies the blood donors (Volunteers) about the blood donation request. It also displays the map plotting the route between the Volunteer and the Requestor's site. It also monitors the distress call handling by ensuring privacy.

In this Paper the related work done by other authors is discussed in section two and in section three the architectural model of the system is explained. Whereas section four describes the various phases involved in providing this service

\section{RELATED WORK}

Few Solutions have been proposed by other authors to contact blood donors during medical emergencies. One such solution is Smart Blood Query (SBQ) mobile system, which selects top five volunteers from the database and sends help request to them. In case of no response from volunteers, the nearby blood bank details is sent to the requester [4]. Other solution 
proposed is that of the Virtual Blood Bank Project, wherein the system also finds a volunteer from the database; residing in the area of the requester and sends their details to the requester [5].

One such solution is an application called Emergency Alert System (EAS), which sends emergency calls to the social contacts of the requester [6]. These above mentioned mobile systems, while requesting for blood donation; considers only static contact location of the blood donors.

Few other solutions like http://www.friends2support.org [3] and http://www.bharatbloodbank.com [7] also register blood donors and show their details to the people requesting for blood help. These above mentioned related work focuses on providing the static location of the blood donors. The above mentioned solutions helps the people to communicate with the blood donors registered in the system based on their registered static addresses and not based on their actual location at the time of need. Also some systems provide the contact details of the blood donors directly to the requester, which may create privacy issues.

The main gap in these solutions is that during medical emergencies, it does not take the actual location of the blood donors into account, while searching them. This proposed system fills up this gap by tracking the actual location of the Blood donor(s) and the system locates the nearest donors at the time of need; based on their actual positioning in the required geographical area. The System does not provide the contact details of the donors directly to the requestors but contacts the blood donors on behalf of the requestors; which maintains the privacy of the donors.

\section{PROPOSED SYSTEM MODEL}

People after facing any road side accidents are taken to the nearest hospitals and most often blood is needed to save the person's life. Hospitals situated in Indian rural areas often may not have sufficient amount of blood especially for the rare blood groups. [8]. Even in the urban areas, where blood banks are situated, supply of blood of rare blood groups may not be available in abundance [9]. However in both these scenarios, there may be a blood donor of required blood group nearby the hospital but not known to the requestor or the hospital. The proposed system helps a patient's relative or hospital's officer to locate nearby volunteers and forward the request for help. The person has to just enter the type of blood group needed in the Citizen's mobile application (CMA) and the system will find the nearest volunteers having the specified blood group and sends the notifications to the Volunteers' Mobile application (VMA); notifying the need of blood donation. The System sends notification to only nearest volunteers by SMS. The volunteer's acknowledgement for the help request is informed to the requestor. The periodic geolocation updates of the volunteer's mobile device; helps the system to find the nearest volunteers at the time of need. The system comprises of client and server application wherein client includes applications like Citizen Mobile Application (CMA), Volunteer Mobile Application and web client. The VMA is responsible to send Location updates of the Volunteer, it fetches the distress call from control server; on arrival of distress call SMS, it sends help confirmation notifications to the control server. The CMA is used by the citizens to send distress call for blood requirement and receives confirmations. The control server records the last geographic location of the volunteer, receives distress calls, searches nearest blood donors and forwards help handling confirmation. The architecture of the system is depicted in figure 1.

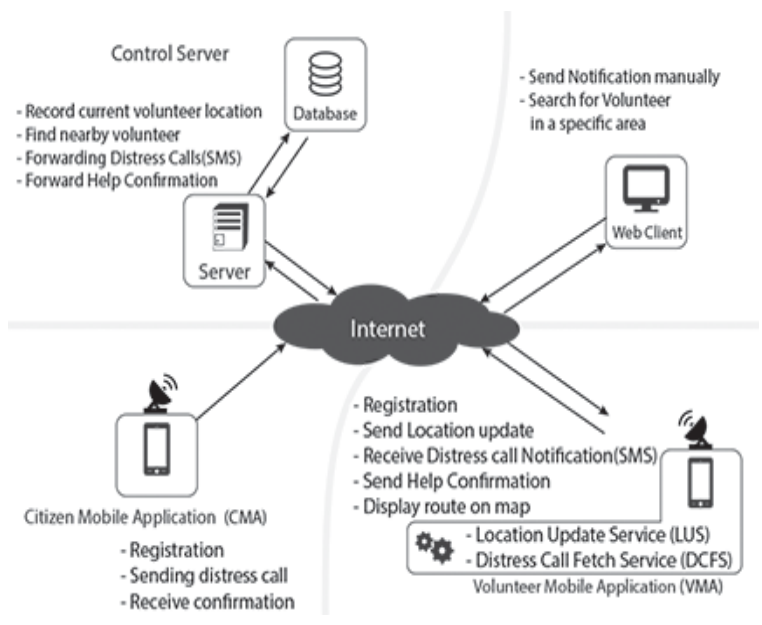

Figure 1: System Architecture Diagram

\section{WORKING OF PROPOSED SYSTEM.}

The system first registers the volunteer and citizens into the system. After registering the volunteer, the system will track the location of the volunteer's mobile device periodically. The citizen can generate a distress call at the time of blood need. The system will get the location of the citizen, based on the current location of the citizen; the system will find the nearest volunteer(s). The system will now send the notification to the nearest volunteers having matching blood group. If the volunteer is ready to help; then the system will show the route to reach the citizen's (Requester) location. These phases are discussed in length in subsection 4.1 to 4.6 .

\subsection{Registering Citizen and Volunteer}

The user needs to register with the system as a volunteer to render help as a blood donor. The Registration process is illustrated in an UML activity diagram shown in figure 2 . The volunteer uses VMA to register with the system. The VMA is currently working for Android [10] smart-phones with version 2.2+. It is successfully tested on Samsung Galaxy 3(android 2.3) and Samsung Galaxy S3 (android 4.2). While registering as a volunteer, the VMA fetches the IMEI (International Mobile Station Equipment Identity) number of the mobile device and the SIM (Subscriber Identity Module) number associated with SIM card inserted in user's mobile device. The IMEI number will be helpful to identify the authenticity of the mobile device. To keep the system spam free, only one user can register from one mobile number. The volunteer can access the VMA through that mobile number only. 


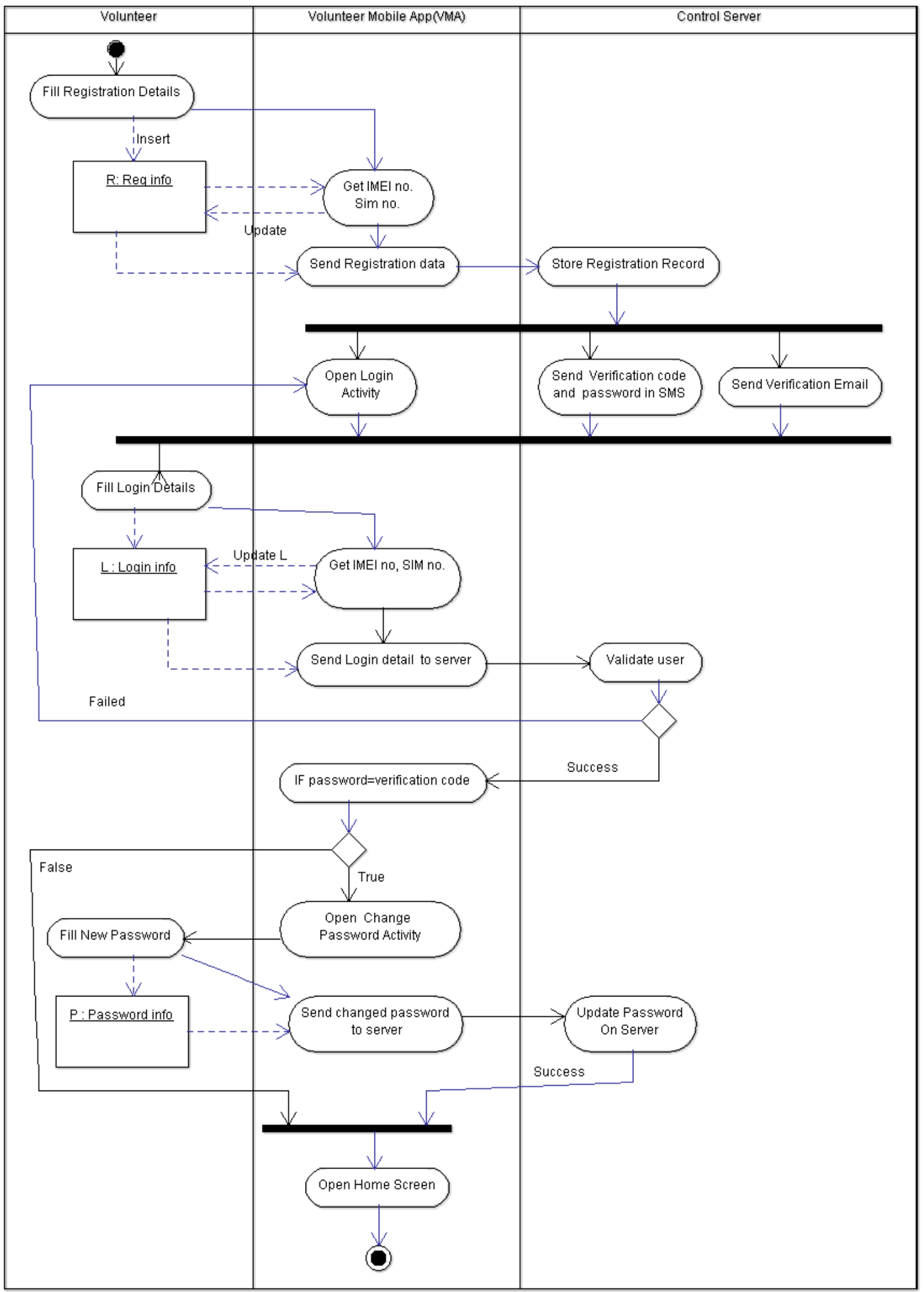

Figure 2: UML Activity Diagram showing Volunteer's Registration Process 
The Registration details are stored on the Control Server into the MySQL [11] database. During Registration Process, control server verifies the mobile number and email of the volunteer by sending a verification code through SMS and a verification URL through email to volunteer's mobile and email account respectively. The SMS is sent through SMS gateway; provided by the SMS gateway service provider to the mobile number given by the volunteer. The verification email contains the URL with unique query string; which is helpful to uniquely identify the volunteer's email id. The user will be registered as a volunteer, finally after conforming the verification code and the verification email.

A person can become a blood donor Volunteer only if ones age is between 18 and 60 years and weighs more than $45 \mathrm{~kg}$. The person should also be disease free and should have haemoglobin count of above $12.5 \mathrm{~g} / \mathrm{dl}$. If the person had suffered from jaundice, then there must a gap of minimum three years. Before donating blood the person should not take any medicine in the last 48 hours and his body temperature and blood pressure should be normal and mainly the person should not be addicted to drugs [12]. The above mentioned test is normally carried out by the Hospital/blood banks. Similarly the Citizens can register to use the system through Citizen's Mobile Application (CMA). The Citizen is the user who will use the system to search Volunteers during need of blood.

\subsection{Tracking Volunteers Location}

Most of the Mobile devices/smart phones are equipped with GPS receivers, which help in getting accurate location of the device. The GPS satellite situated in the space continuously transmits data containing the location and time details. When the mobile device requests for the location then its GPS receiver receives the data sent from GPS Satellite and displays the current location.

Location can also be obtained by mobile cell tower. The cell tower sends its location to the mobile device at first call and as the request increases; the location becomes more accurate. The more number of cell towers around the mobile device will lead to find more accurate location in less time.

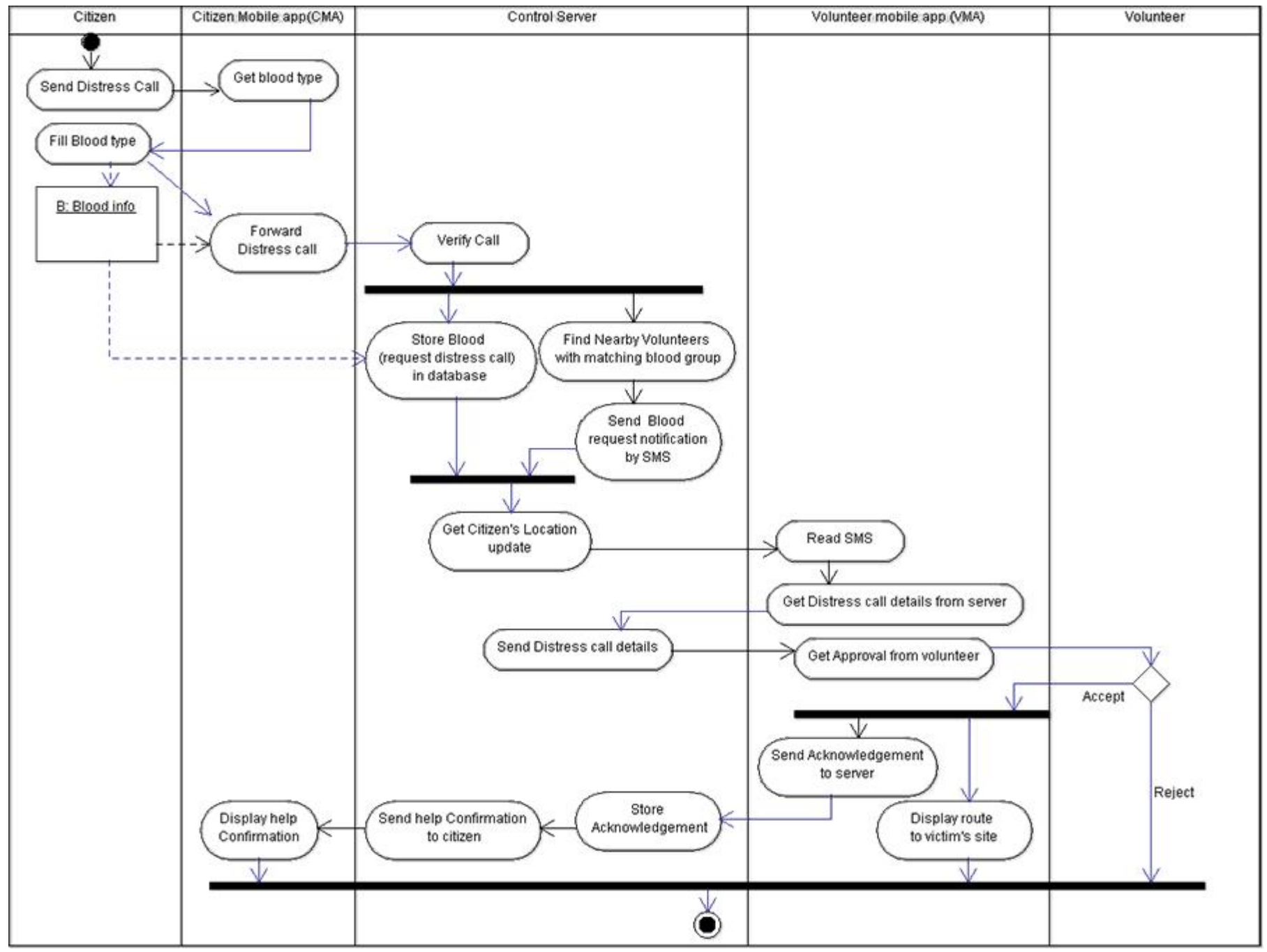

Figure 3: Activity diagram showing the process of sending Distress Call (Help Call) 
The system periodically tracks the current location of the registered Blood Donors (volunteers) using GPS (if available) or Mobile Network Location. Both these services are integrated in VMA. The VMA will keep the Location Updater Service (LUS) running in background. The Volunteer LUS will send data containing current geo-location (latitude, longitude and altitude) of the volunteer's Mobile Device to the control server. The control server will record last Geolocation of the volunteer into the database along with the timestamp.

Sending frequent current geo-location updates of the volunteer to the server has two issues: privacy of the person, as the person's geo-location is periodically sent to the server, and the more data and power consumption of the volunteer's mobile device.

The system is designed to overcome the privacy issue regarding the periodic geo-location update. To preserve privacy of the volunteer, the location update service can be stopped temporarily. To avoid the large amount of data consumption and the power consumption of the Volunteer's mobile device, the LUS will get the current geo-location of the Volunteer's Mobile device through GPS or the Mobile Network and verifies it with the previous updated location information of the Volunteer's Mobile device. The LUS will send the location update to the control server, only when the difference between the current and previous location of the Volunteer's Mobile device is more than K Kilometres. The value of $\mathrm{K}$ variable depends on the geographical terrain in which the volunteer resides. The value of $\mathrm{K}$ will be larger in flat terrains and lower in mountainous terrains. This helps in reducing the power and packet data consumption of the Volunteer's Mobile device. The Volunteer can also set the periodicity to send location updates in the LUS. This will help in reducing the power consumption of the mobile device.

\subsection{Sending Distress (Help) Call}

The CMA can be used to place a distress call for blood requirement, when a citizen is in need of blood in emergency but cannot find enough supply of blood for a particular blood group in the Blood bank or at the Hospital. In this type of circumstances the citizen can avail the facility of communicating the help request to nearby blood donor volunteers by pressing the blood request button (distress call) in the CMA. The process of sending a distress call is illustrated in an UML activity diagram shown in figure 3 . The CMA asks the citizen to enter the patient's blood group. The CMA will then send the blood group and the current geolocation of the requestor's mobile device to the control server. The patient's (requestor's) location is obtained through GPS or the Mobile Network. The control server will receive the blood request call and authenticate it. The authentication process is done by confirming that the SIM number and IMEI number received in the distress call are registered. After authenticating the distress help call, the control server will find the nearest Volunteers in the vicinity of $\mathrm{N}$ Kilometres (Zone I) around the patient's site. The system will then send help notifications to the volunteers found in that Zone.

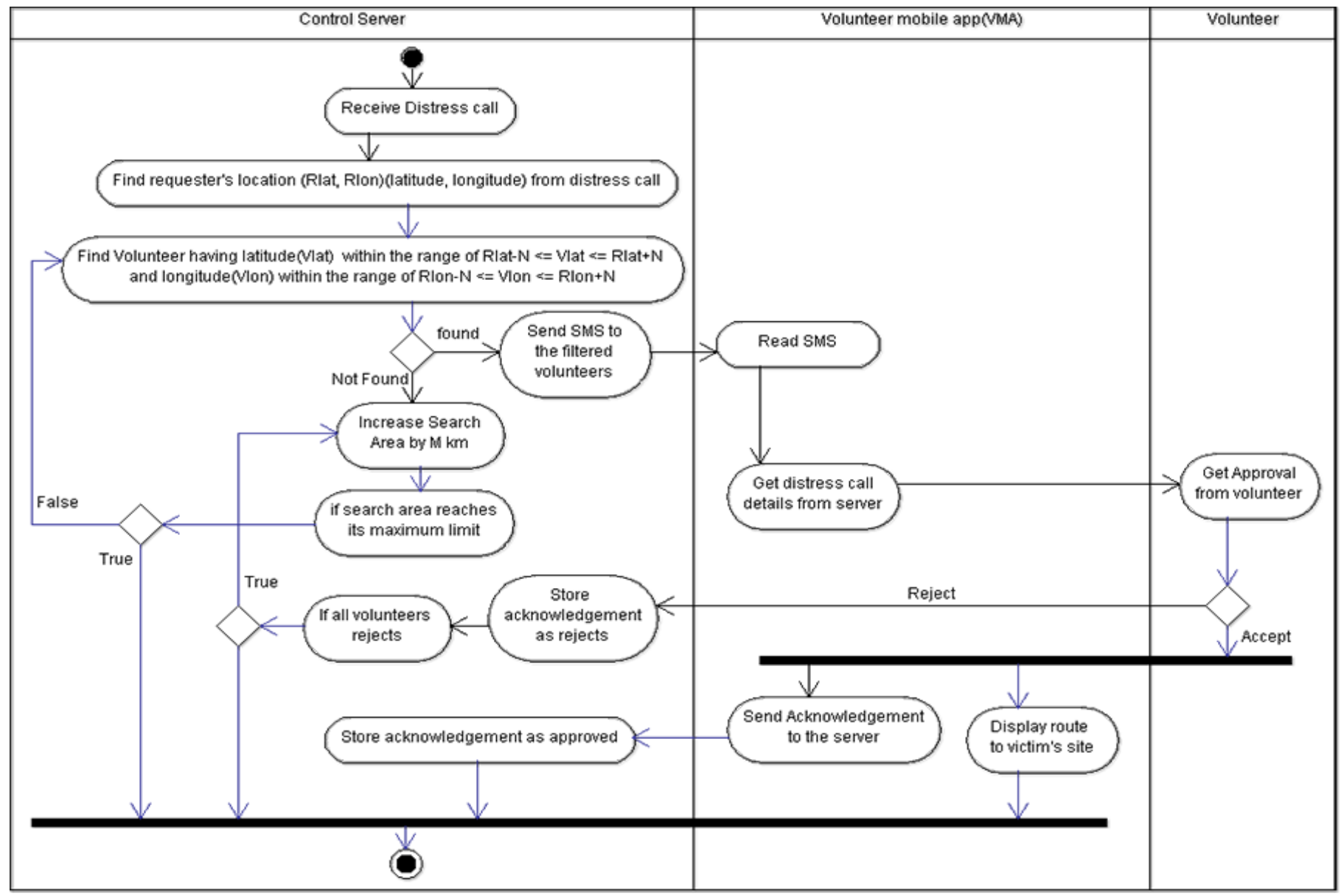

Figure 4: UML Activity diagram for finding nearby volunteers 


\subsection{Finding nearest Volunteers}

When the System receives a help call; it first filters the Volunteers present in the area of $\mathrm{N}$ kilometres around the requester's location $\left(\mathrm{R}_{\text {lat }}, \mathrm{R}_{\text {lon }}\right)$. The process of finding nearest volunteers is illustrated using UML activity diagram shown in figure 4 . The system selects the volunteers whose latitude $\left(\mathrm{V}_{\text {lat }}\right)$ is within the range of $\mathrm{R}_{\text {lat }}-\mathrm{N}<=\mathrm{V}_{\text {lat }}<=\mathrm{R}_{\text {lat }}+\mathrm{N}$ and longitude $\left(\mathrm{V}_{\text {lon }}\right)$ is within the range $\mathrm{R}_{\text {lon }}-\mathrm{N}<=\mathrm{V}_{\text {lon }}<=\mathrm{R}_{\text {lon }}$ $+\mathrm{N}$, which virtually creates a search area (Zone I) as shown in figure 5. The above mentioned formula requires less searching time for finding nearby volunteers. After the system finds the volunteers in Zone I, it filters them by the patient's matching blood groups and sends help notification SMS to these volunteers.

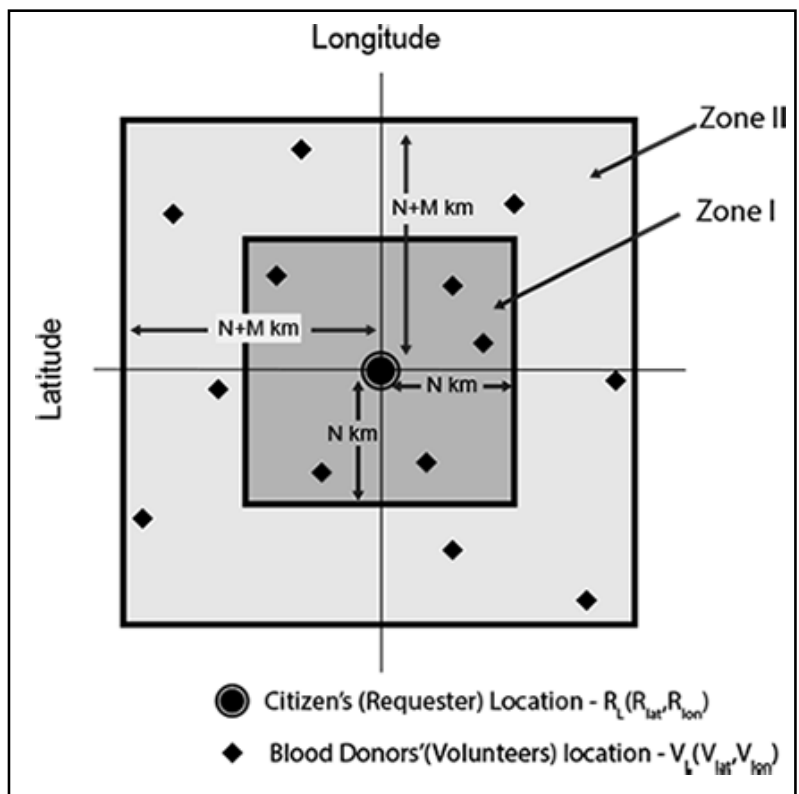

Figure 5: search zone to find volunteers (blood donors)

When the system does not get acknowledgement from any volunteer or none of volunteers are found around in the $\mathrm{N}$ kilometres area of requester's location, than in that case the search area is broadened to N+M kilometres. The search area is recursively incremented by $\mathrm{M}$ kilometres till a volunteer is found.

The system stops finding the volunteers when the search area reaches its maximum limit. e.g. the initial searching area $\mathrm{N}$ can be 5 Kilometres and incremental value $M$ can be 2 Kilometres. The Value of $\mathrm{N}$ and $\mathrm{M}$ variables is dependent on the geographic terrain in which the requestor is located.

\subsection{Notifying Volunteers about Distress (Help) Call.}

The system after finding the nearest volunteers will send them a SMS. The SMS is sent using the SMS Gateway Service Provider as stated earlier. After the registration process as mentioned in section 4.1, the VMA registers a Broadcast Receiver, which is enabled to read all the incoming SMS. A Broadcast Receiver (short receiver) is an Android component which allows you to register for system or application events [13]. When the SMS from the system is received on the VMA, the Broadcast Receiver will read and identify the SMS and then will start a Distress Call Fetch Service (DFCS). DFCS will send volunteer id to the server. The Control server will return the distress calls linked with the volunteer. The details received by the VMA will be in JSON (JavaScript
Object Notation) format. JSON is a light-weighted data exchange format, which is comprised of key-value pair. It makes JSON faster in exchanging data between systems [14]. After receiving the distress call details, DFCS will generate a push notification to notify the volunteer about the distress call. The VMA sends an acknowledgement to the control server when the volunteer accepts the help notification. The control server records the acknowledgement and forwards the confirmation to the Requestor through SMS.

\subsection{Displaying Route from Volunteer's Location to Victim's Site}

Once the volunteer clicks on the distress call notification, the volunteer is displayed the Map which shows the route to reach the patient's (requestor's) Site. The Google Map API is used to draw the path on Google map [15]. A direction path is drawn from the volunteer's current location to the requestor's current location, which helps the volunteer to reach the patient's site (refer figure 6). The VMA keeps updating the Volunteer's current location in the Map. The patient's current location also keeps on updating because the patient might be shifted or moved to another place or Hospital. Location of Volunteer and patient is obtained using GPS or Cell Tower of the mobile service provider as mentioned in subsection 4.2.

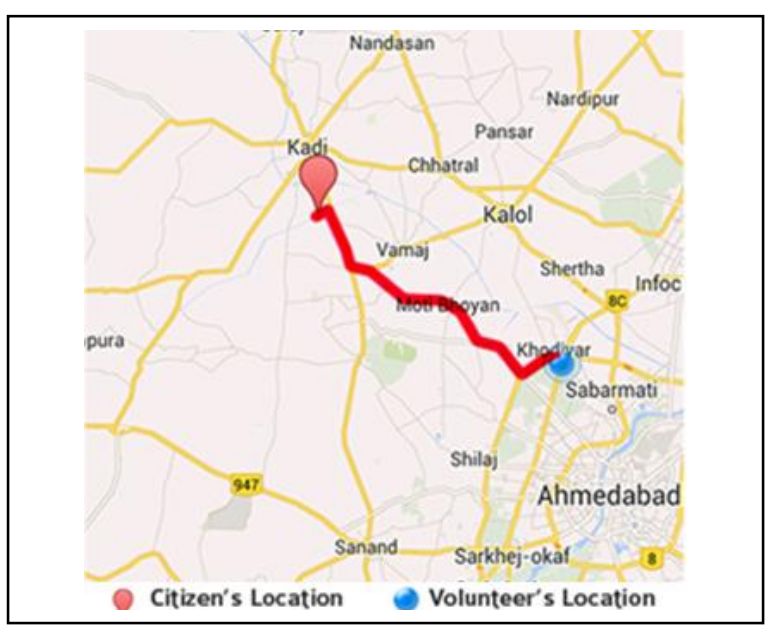

Figure 6: Route information plotted on Google Map to reach Requestor' Site from the volunteer's current site

\section{CONCLUSION}

The system provides a better, faster and effective way for the citizens to communicate with the blood donor for blood requirement in times of medical emergency. Finding the nearest volunteers helps in increasing the chance of saving the patient's life especially in rural areas, where hospitals and blood banks are at a far distance. People living in places where blood banks are not available; can use this System in medical emergency situations. It is even helpful in different type of geographical terrains to provide aid in saving life.

In future, the system will analyze the volunteer's location travel pattern and on this basis; the volunteer will be notified for a distress call even if his LUS is disabled. Present system is dependent on GPS service and uses packet data of mobile device to send and receive distress call. In future, CMA and VMA will send or receive distress calls through SMS even when the Packet Data connection is not available. 


\section{REFERENCES}

[1] A. Chakrabarty, "India faces huge blood donation deficit," Zee News, p. 1, 14 June 2012.

[2] C. D. S. Control, "NUMBER OF LICENSED BLOOD BANKS IN THE COUNTRY UP TO November 2012," 2012.

[3] "Friends2Support," [Online]. Available: http://www.friends2support.org.

[4] K. A. A. S. H. A. B. S. I. A. Muhammad Sajidur Rahman, "Smart Blood Query," 2011.

[5] P. B. a. S. K. Ramesh Singh, "Smart Phones to the Rescue," 2007.

[6] A. C. S. A. G.-S. Michael Ovelg"onne, "Social Emergency Alert Service - A Location-Based," 2010.

[7] "Bharat Blood Bank," [Online]. Available: http://www.bharatbloodbank.com.
[8] S. Reporter, "Only 81 Blood banks in Rural areas in india," The Hindu, 16 December 2012.

[9] K. Dixit, "Now, a directory of blood donors," The Times Of India, p. 1, 1 November 2011.

[10] "Android Developers," Google, [Online]. Available: http://www.developer.android.com/.

[11] "MySQL," Oracle, [Online]. Available: http://www.mysql.com.

[12] S. S. N. K. R. R. MUHAMMAD ARIFI, “AUTOMATED ONLINE BLOOD BANK,” 2012.

[13] L. Vogel, “Android BroadcastReceiver Tutorial”.

[14] "JSON," [Online]. Available: http://www.json.org.

[15] "Maps API, Google Developers," Google, [Online]. Available: https://developers.google.com/maps. 